\title{
Sarcoidosis-Associated Fibrosing Mediastinitis with Resultant Pulmonary Hypertension: A Case Report and Review of the Literature
}

\author{
Rebecca L. Toonkel $^{a} \quad$ Alain C. Borczuk ${ }^{b}$ Gregory D. Pearson ${ }^{c}$ Evelyn M. Horn ${ }^{d}$ \\ Byron M. Thomashow ${ }^{\mathrm{a}}$
}

aDivision of Pulmonary, Allergy, and Critical Care Medicine, and Departments of ${ }^{\mathrm{b}}$ Pathology, ${ }^{\mathrm{C}}$ Radiology and

${ }^{\mathrm{d} D i v i s i o n}$ of Cardiology, Columbia University Medical Center, New York, N.Y., USA

\section{Established Facts}

- Pulmonary hypertension is a common complication of advanced sarcoidosis resulting from fibrosis with chronic hypoxia or destruction of small vessels, extrinsic compression of pulmonary arteries by granulomatous disease or lymphadenopathy, and granulomatous vasculitis.

\section{Novel Insights}

- Sarcoidosis-associated pulmonary hypertension may also develop as a result of fibrosing mediastinitis with pulmonary venous compression.

- Sarcoidosis should be considered in the differential diagnosis of patients with unexplained fibrosing mediastinitis and pulmonary hypertension.

\section{Key Words}

Sarcoidosis · Hypertension, pulmonary · Mediastinitis, fibrosing

\begin{abstract}
Pulmonary hypertension, a common manifestation of advanced sarcoidosis, is thought to result from fibrosis with chronic hypoxia and destruction of small vessels, extrinsic compression of pulmonary arteries, or granulomatous vasculitis. We report a case of sarcoidosis-associated pulmonary hypertension due to fibrosing mediastinitis. Our patient pre-
\end{abstract}

sented with cough and dyspnea on exertion and was found to have pulmonary artery enlargement, pulmonary venous compression, and mediastinal soft tissue enhancement on magnetic resonance imaging. Pulmonary hypertension was confirmed by right heart catheterization and sarcoidosis was diagnosed by histologic examination of tissue obtained at mediastinoscopy. Treatment with steroids resulted in decreased pulmonary artery pressures as well as symptomatic improvement. While pulmonary hypertension is a common complication of sarcoidosis, fibrosing mediastinitis is an unusual etiology that should be considered by clinicians.

Copyright ๑ 2009 S. Karger AG, Basel

\section{KARGER}

Fax +41 613061234 E-Mail karger@karger.ch www.karger.com
(C) 2009 S. Karger AG, Basel

0025-7931/10/0794-0341\$26.00/0

Accessible online at:

www.karger.com/res
Byron M. Thomashow, MD, FCCP

$161 \mathrm{Ft}$. Washington Avenue, Ste 311

New York, NY 10032 (USA)

Tel. +1 212305 5261, Fax +1 2123421856

E-MailBmt1@columbia.edu 


\section{Introduction}

Pulmonary hypertension occurs in up to $28 \%$ of patients with sarcoidosis [1-6] and is even more prevalent with advanced disease $[2,7]$. Common etiologies of pulmonary hypertension include fibrosis with chronic hypoxia or destruction of small vessels $[1,2,4,5,7]$, extrinsic compression of pulmonary arteries by granulomatous disease $[8,9]$ or lymphadenopathy $[10]$, and granulomatous vasculitis [11-13]. We report a patient presenting with pulmonary hypertension due to sarcoidosis-associated fibrosing mediastinitis.

\section{Case Report}

A 45-year-old man presented after experiencing high-altitude pulmonary edema. Two years prior, he noted cough and sought treatment because of increasing dyspnea while jogging. Pulmonary function testing (PFTs) reportedly showed an $\mathrm{FEV}_{1} / \mathrm{FVC} \mathrm{ra-}$ tio of $70 \%$ and preserved diffusing capacity for carbon monoxide and total lung capacity. The chest X-ray was normal. He was diagnosed with asthma and was prescribed fluticasone/salmeterol without relief. Then, while hiking, he became acutely short of breath. A CT angiogram (CTA) revealed no pulmonary emboli, but showed scattered parenchymal densities and small $(<1 \mathrm{~cm})$ nodules, mediastinal lymphadenopathy, and increased mediastinal soft tissue. His symptoms resolved upon return to sea level with corticosteroids. He returned home and was referred to our clinic.

He had no past medical history, was taking no medications, had never smoked cigarettes, and had no relevant family history. Vital signs were: blood pressure 140/90 mm Hg; heart rate 84; respiratory rate 16 , and $\mathrm{SaO}_{2}$ at room air was $96 \%$ at rest and $94 \%$ with exercise. He appeared well, the jugular venous pressure was not elevated and the lungs were clear bilaterally. The heart was regular without murmurs or gallops, but there was an exaggerated splitting of the second heart sound with a prominent pulmonic component and a palpable right ventricular heave. The remainder of the examination was unremarkable.

Laboratory evaluations revealed normal blood counts, electrolytes, kidney and liver function. Normal serologies included erythrocyte sedimentation rate, rheumatoid factor, antinuclear antibody, antineutrophil cytoplasmic antibody, quantitative immunoglobulins, and a negative hypersensitivity pneumonitis allergen panel. Also negative were antibodies to HIV, histoplasma antigen and antibodies, and a purified protein derivative. Repeat pulmonary function testings revealed $\mathrm{FEV}_{1} / \mathrm{FVC} 56 \%$ and preserved diffusing capacity for carbon monoxide and lung volumes. Repeat CTA confirmed the prior findings as well as enlargement of the pulmonary artery $(3.4 \mathrm{~cm}$; fig. 1$)$. Magnetic resonance angiography (MRA) showed lymphadenopathy and diffuse soft tissue enhancement suggestive of fibrosing mediastinitis encasing and narrowing the pulmonary veins, most prominent on the left (fig. 2, 3). Pulmonary hypertension was confirmed by transthoracic echocardiogram (estimated pulmonary artery systolic pressure $60 \mathrm{~mm} \mathrm{Hg}$ ) and showed mild dilation and hypokinesis of the right ventricle but no left heart abnormalities. Cardiac MRI revealed epicardial scarring involving $1-25 \%$ of the myocardial thickness at the inferior wall, but no wall motion abnormalities, normal chamber sizes, an ejection fraction of $51 \%$, and a mildly dilated main pulmonary artery. On right heart catheterization, right atrial pressure was $20 \mathrm{~mm} \mathrm{Hg}$, pulmonary artery pressure was $62 / 30$ (mean 44$) \mathrm{mm} \mathrm{Hg}$, pulmonary capillary wedge pressure (PCWP) measured on the left was $24 \mathrm{~mm} \mathrm{Hg}$, and pulmonary vascular resistance (PVR) was low at 2.6, supporting a diagnosis of pulmonary venous hypertension. Pulmonary artery oxygen saturation was elevated at $80 \%$ and cardiac output was 8.3 .

Mediastinoscopy revealed diffuse fibrosis and multiple firm pretracheal nodes. Biopsy specimens showed non-necrotizing granulomas surrounded by abundant fibrosis and special stains and cultures for fungi and acid fast bacilli were negative. Based on these results, the patient was diagnosed with sarcoidosis and pulmonary hypertension was attributed to fibrosing mediastinitis with pulmonary vein compression. Treatment with prednisone was initiated at $60 \mathrm{mg} / \mathrm{day}$ and repeat echocardiogram at 8 weeks showed a reduced right ventricular systolic pressure (45 $\mathrm{mm} \mathrm{Hg}$ ). Repeat MRA at 6 months showed persistent mediastinal soft tissue enhancement, but decreased pulmonary venous compression (fig. 2). Finally, repeat right heart catheterization at 1 year showed improved right atrial pressure (10 from $20 \mathrm{~mm} \mathrm{Hg}$ ), pulmonary artery pressure (mean 34 from $44 \mathrm{~mm} \mathrm{Hg}$ ), and PCWP (16 from $24 \mathrm{~mm} \mathrm{Hg}$ ), with a similar PVR (4.3 from 2.6). Within weeks of initiating treatment, the patient noted improved exercise tolerance. Prednisone was slowly tapered over 1 year.

\section{Discussion and Literature Review}

Based on our review, this is the first reported case of sarcoidosis-associated fibrosing mediastinitis with pulmonary venous compression and resultant pulmonary hypertension. While fibrosing mediastinitis is not common with sarcoidosis, pulmonary hypertension occurs in up to $28 \%$ of patients $[1-5,14]$. However, because this estimate reflects the prevalence at referral centers, it may be biased $[1,6]$. The estimated 5-year survival for patients with sarcoidosis-associated pulmonary hypertension is just 59\% [7]. These patients are more likely to be listed for transplant than those with sarcoidosis alone [6], require more supplemental oxygen, have poorer functional status, and have increased mortality while awaiting transplant $[10,15]$.

Etiologies of pulmonary hypertension with sarcoidosis include fibrosis with chronic hypoxia or destruction of small vessels $[1,2,5,14]$, extrinsic compression of pulmonary arteries by granulomatous disease $[8,16]$ or lymphadenopathy [7], and granulomatous vasculitis [9, $11,13]$ which occasionally mimics pulmonary veno-occlusive disease $[7,12,17]$. These etiologies were unlikely in our patient as he had no radiographic evidence of pa- 

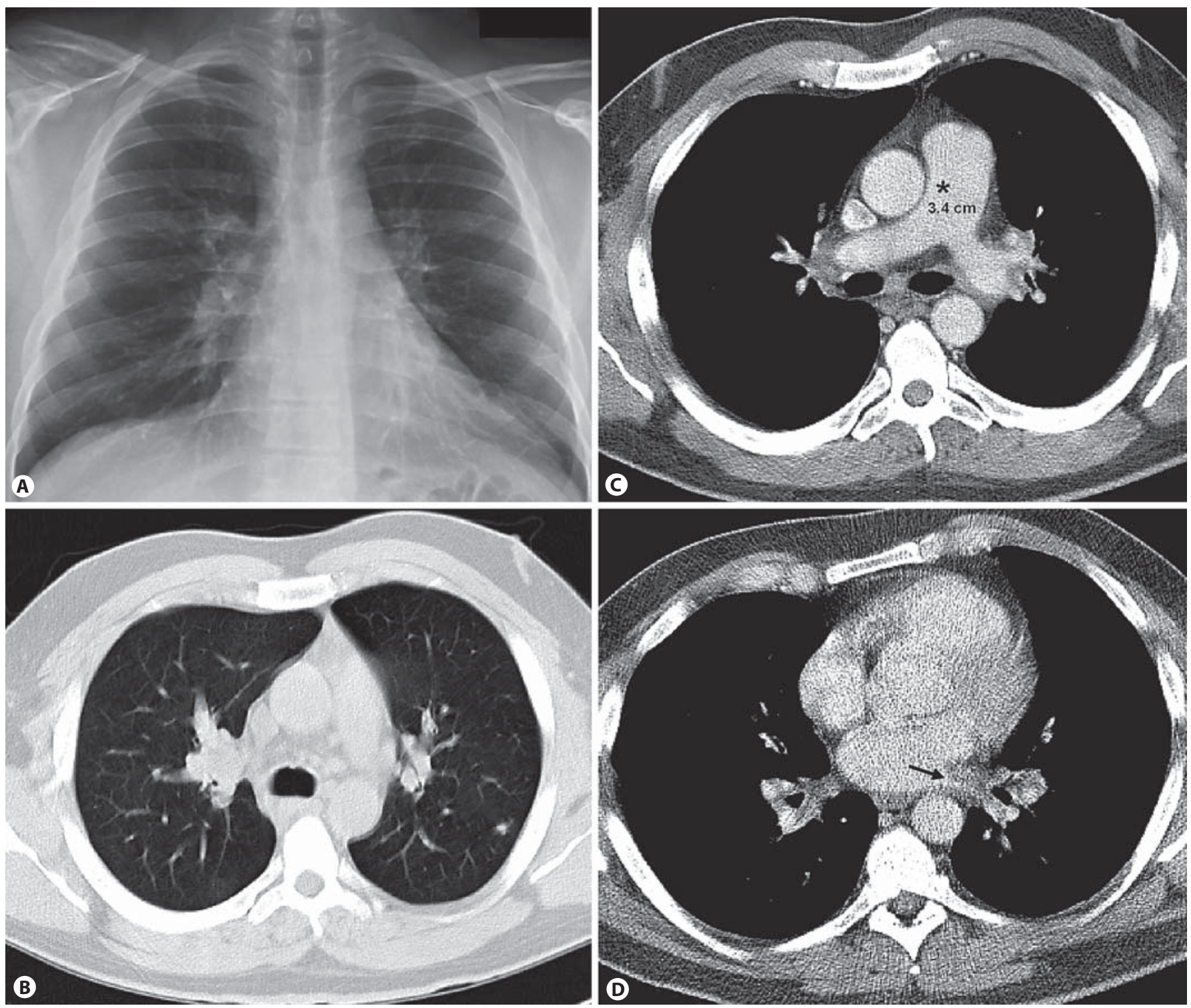

Fig. 1. A PA chest radiograph showing mild hilar prominence. B Non-contrast CT showing multiple small nodules, mediastinal lymphadenopathy, and increased mediastinal soft tissue. C, D CTA showing enlargement of the pulmonary artery (asterisk) and narrowing of the left inferior pulmonary vein (arrow).

renchymal fibrosis, normal resting oxygen saturation, no arterial compression by MRA and no perfusion abnormalities by CTA. However, because the PCWP was measured unilaterally, it is impossible to completely rule out a contribution from any of these factors.

Less common etiologies of sarcoidosis-associated pulmonary hypertension include portal-pulmonary hypertension $[18,19]$ and vasoconstriction due to the elaboration of vasoactive factors [20]. Our patient had normal liver function and normal PVR making these etiologies unlikely. While the PCWP was elevated, because the left heart was normal by echocardiography, this more likely reflects pulmonary venous hypertension than myocardial dysfunction. Also, while cardiac MRI noted mild epicardial scarring, this involved a limited thickness of the myocardium in a small portion of the wall and did not affect left heart size or function, making primary cardiac sarcoid unlikely. An extensive workup for other causes of non-sarcoidosis-related pulmonary hypertension was unrevealing, leaving mediastinal fibrosis with 

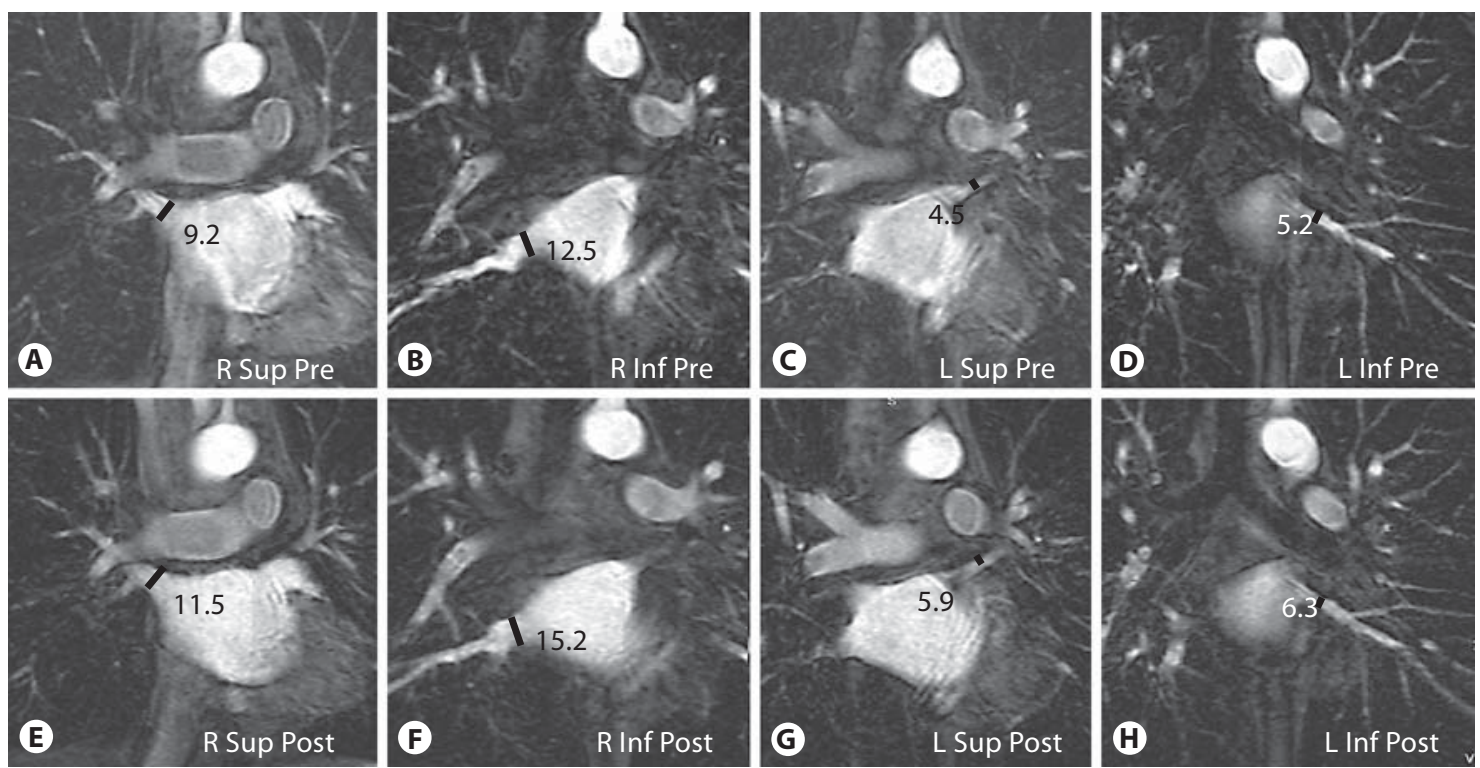

Fig. 2. A-D Magnetic resonance angiography showing soft tissue compression of the pulmonary veins prior to treatment. E-H Repeat imaging after treatment showed persistent but improved narrowing of the pulmonary veins.

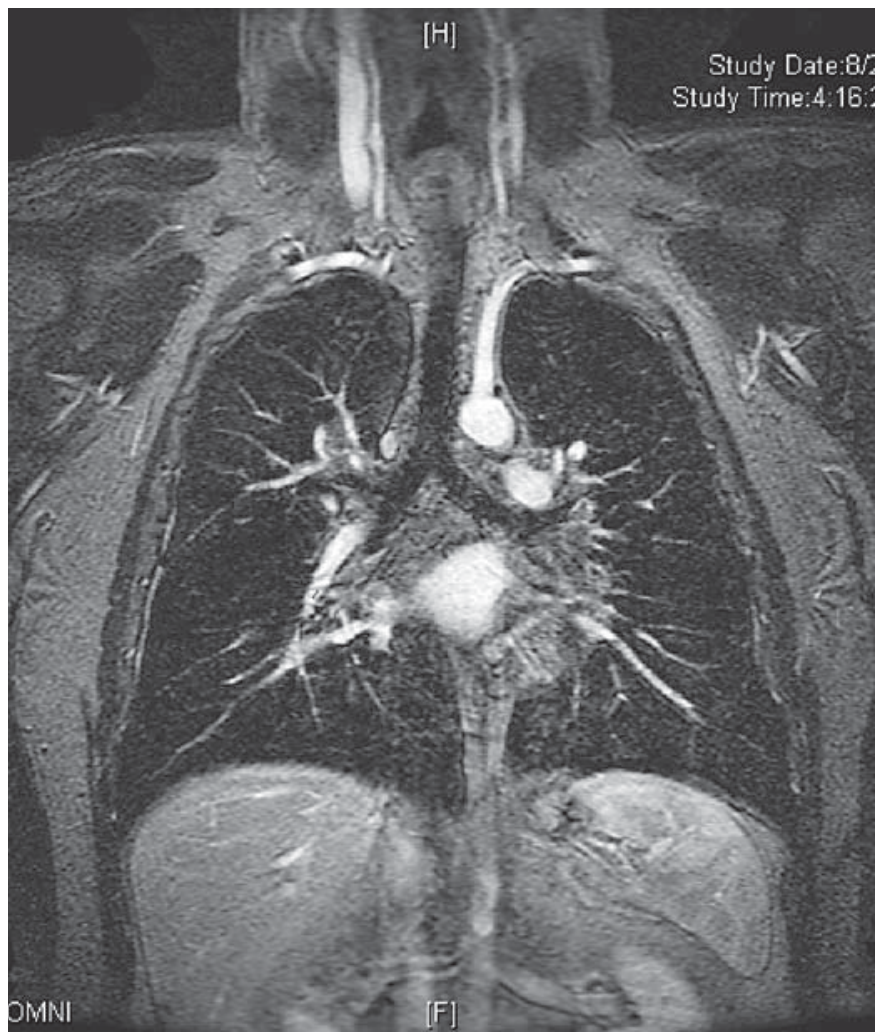

Fig. 3. Magnetic resonance angiography showing soft tissue enhancement throughout the mediastinum. resultant pulmonary venous compression the most likely etiology.

Previously, sarcoidosis-associated pulmonary hypertension was attributed only to pulmonary fibrosis leading to chronic hypoxia and small vessel destruction. Accordingly, its most commonly cited clinical predictors include advanced Scadding stage $[3,21]$ and low oxygen saturation [3]. However, studies have shown that neither of these factors accurately predicts the incidence or severity of pulmonary hypertension $[7,21,22]$. This is supported not only by a higher incidence of pulmonary hypertension in sarcoidosis than that seen in other destructive lung diseases [23], but also by the fact that the mean pulmonary artery pressure of patients awaiting transplant is significantly higher for patients with sarcoidosis than those with idiopathic pulmonary fibrosis [24]. Other potential markers, such as spirometry, lung volumes, and diffusing capacity, are equally unreliable $[6,7,10,21]$. Similarly, neither lymph node enlargement nor thickening of bronchovascular bundles by CT accurately predicts pulmonary hypertension [3]. Perhaps the difficulty in identifying reliable predictors of pulmonary hypertension in sarcoidosis reflects the fact that no studies to date have stratified patients by underlying etiology. Because there are multiple etiologies of pulmonary hypertension in sarcoidosis, predictors may vary with etiology. 
Similarly, treatment efficacy may depend upon etiology and the failure to differentiate patients on this basis may explain our relative lack of success in treating sarcoidosis-associated pulmonary hypertension. Optimal treatment is unknown but is currently the subject of ongoing research. Several small studies and case series, including one published in this journal, have reported variable responses to vasodilator treatments [23, 25-27]. While these studies suggest that at least a subset of patients may have a clinical response, the question must be addressed more rigorously by large randomized controlled trials.

As with vasodilators, response to corticosteroids is also variable. While several small series suggest improve- ment $[11,28]$, others have found mixed responses $[2,7]$ or no benefit at all $[8,13,17]$. Our patient improved with steroids as evidenced by improved hemodynamics and decreased pulmonary venous compression. While anecdotal, this suggests that pulmonary hypertension due to diffuse mediastinal fibrosis may be steroid-responsive. As with vasodilators, randomized controlled trials will be necessary to definitively assess the role of steroids. Such studies should also stratify patients by etiology in order to identify accurate predictors of response. This will help to tailor therapies and thus improve the efficacy of treatment regimens prescribed for this complicated disease.

\section{References}

1 Gluskowski J, Hawrylkiewicz I, Zych D, Wojtczak A, Zielinski J: Pulmonary haemodynamics at rest and during exercise in patients with sarcoidosis. Respiration 1984;46: 26-32.

-2 Gluskowski J, Hawrylkiewicz I, Zych D, Zielinski J: Effects of corticosteroid treatment on pulmonary haemodynamics in patients with sarcoidosis. Eur Respir J 1990;3:403407.

3 Handa T, Nagai S, Miki S, Fushimi Y, Ohta $\mathrm{K}$, Mishima M, et al: Incidence of pulmonary hypertension and its clinical relevance in patients with sarcoidosis. Chest 2006;129: 1246-1252.

4 Mitchell DN, Scadding JG: Sarcoidosis. Am Rev Respir Dis 1974;110:774-802.

5 Rizzato G, Pezzano A, Sala G, Merlini R, Ladelli L, Tansini G, et al: Right heart impairment in sarcoidosis: haemodynamic and echocardiographic study. Eur J Respir Dis 1983;64:121-128.

-6 Shorr AF, Helman DL, Davies DB, Nathan SD: Pulmonary hypertension in advanced sarcoidosis: epidemiology and clinical characteristics. Eur Respir J 2005;25:783-788.

-7 Nunes H, Humbert M, Capron F, Brauner M Sitbon O, Battesti JP, et al: Pulmonary hypertension associated with sarcoidosis: mechanisms, haemodynamics and prognosis. Tho$\operatorname{rax} 2006 ; 61: 68-74$.

-8 Damuth TE, Bower JS, Cho K, Dantzker DR: Major pulmonary artery stenosis causing pulmonary hypertension in sarcoidosis. Chest 1980;78:888-891.

9 Levine BW, Saldana M, Hutter AM: Pulmonary hypertension in sarcoidosis. A case report of a rare but potentially treatable cause. Am Rev Respir Dis 1971;103:413-417.
10 Shorr AF, Davies DB, Nathan SD: Predicting mortality in patients with sarcoidosis awaiting lung transplantation. Chest 2003;124: 922-928.

-11 Davies J, Nellen M, Goodwin JF: Reversible pulmonary hypertension in sarcoidosis. Postgrad Med J 1982;58:282-285.

12 Hoffstein V, Ranganathan N, Mullen JB: Sarcoidosis simulating pulmonary veno-occlusive disease. Am Rev Respir Dis 1986;134: 809-811.

13 Smith LJ, Lawrence JB, Katzenstein AA: Vascular sarcoidosis: a rare cause of pulmonary hypertension. Am J Med Sci 1983;285:3844.

14 Battesti JP, Georges R, Basset F, Saumon G: Chronic cor pulmonale in pulmonary sarcoidosis. Thorax 1978;33:76-84.

15 Arcasoy SM, Christie JD, Pochettino A, Rosengard BR, Blumenthal NP, Bavaria JE, et al: Characteristics and outcomes of patients with sarcoidosis listed for lung transplantation. Chest 2001;120:873-880.

16 Tayal S, Voelkel NF, Rai PR, Cool CD: Sarcoidois and pulmonary hypertension - a case report. Eur J Med Res 2006;11:194-197.

17 Portier F, Lerebours-Pigeonniere G, Thiberville L, Dominique S, Tayot J, Muir JF, et al: Sarcoidosis simulating a pulmonary venoocclusive disease (in French). Rev Mal Respir 1991;8:101-102.

18 Salazar A, Mana J, Sala J, Landoni BR, Manresa F: Combined portal and pulmonary hypertension in sarcoidosis. Respiration 1994; 61:117-119.

19 Cornet A, Barbier JP, Debesse B, Even P, Carnot F, Neveu D: Sarcoidosis and portal and pulmonary arterial hypertension: a case report (author's transl) (in French). Ann Med Interne (Paris) 1980;131:431-434.
20 Barst RJ, Ratner SJ: Sarcoidosis and reactive pulmonary hypertension. Arch Intern Med 1985;145:2112-2114.

-21 Sulica R, Teirstein AS, Kakarla S, Nemani N, Behnegar A, Padilla ML: Distinctive clinical, radiographic, and functional characteristics of patients with sarcoidosis-related pulmonary hypertension. Chest 2005;128:14831489.

22 Emirgil C, Sobol BJ, Herbert WH, Trout K: The lesser circulation in pulmonary fibrosis secondary to sarcoidosis and its relationship to respiratory function. Chest 1971;60:371378.

23 Fisher KA, Serlin DM, Wilson KC, Walter RE, Berman JS, Farber HW: Sarcoidosis-associated pulmonary hypertension: outcome with long-term epoprostenol treatment. Chest 2006;130:1481-1488.

24 Shorr AF, Davies DB, Nathan SD: Outcomes for patients with sarcoidosis awaiting lung transplantation. Chest 2002;122:233-238.

- 25 Preston IR, Klinger JR, Landzberg MJ, Houtchens J, Nelson D, Hill NS: Vasoresponsiveness of sarcoidosis-associated pulmonary hypertension. Chest 2001;120:866872.

26 Milman N, Burton CM, Iversen M, Videbaek $\mathrm{R}$, Jensen CV, Carlsen J: Pulmonary hypertension in end-stage pulmonary sarcoidosis: therapeutic effect of sildenafil? J Heart Lung Transplant 2008;27:329-334.

27 Foley RJ, Metersky ML: Successful treatment of sarcoidosis-associated pulmonary hypertension with bosentan. Respiration 2008; 75 : 211-214.

28 Rodman DM, Lindenfeld J: Successful treatment of sarcoidosis-associated pulmonary hypertension with corticosteroids. Chest 1990;97:500-502. 